\title{
Dynamics of Microbial Communities on Eggshells and on Nest Materials During Incubation in the Oriental Tit (Parus Minor)
}

Hokyung Song

Seoul National University

Keesan Lee

Seoul National University

Injae Hwang

Seoul National University

Eunjeong Yang

Seoul National University

Jungmoon $\mathrm{Ha}$

Seoul National University

Woojoo Kim

Seoul National University

Sungjin Park

Seoul National University

Hyunjoon Cho

Korea Polar Research Institute

Jae Chun Choe

Ewha Womans University

Sang-im Lee ( $\nabla$ sangim@dgist.ac.kr)

DGIST: Daegu Gyeongbuk Institute of Science and Technology https://orcid.org/0000-0001-7808-7140

Piotr G. Jablonski

Seoul National University

\section{Research Article}

Keywords: Parus minor, Eggshell, Nest material, Microbial community, Incubation, Dynamics

Posted Date: June 9th, 2021

DOI: https://doi.org/10.21203/rs.3.rs-585254/v1 
License: (c) (i) This work is licensed under a Creative Commons Attribution 4.0 International License. Read Full License 


\section{Abstract}

Eggshell microbial communities may affect hatching success and nestling's condition. Nest materials are in direct contact with the eggshells, but the relationships with the eggshell microbiome during incubation have not been fully elucidated. Here, we characterize eggshell and nest-material microbial communities and their changes during incubation in the Oriental Tit (Parus minor). Microbial communities on the nest material were relatively stable and remained distinct from the eggshell communities, and had higher diversity and greater phylogenetic clustering compared to the eggshell communities from the same nest, resulting in lower phylogenetic turnover rate of nest material microbiome during incubation than expected by chance. While the species diversity of both communities did not change during incubation, we found significantly greater changes in the structure of microbial communities on the eggshell than on the nest material. However, eggshell microbiome remained distinct from nest material microbiome, suggesting independent dynamics between the two microbiomes during incubation. We detected an increase in the relative abundance of several bacterial taxa on the eggshell that likely come from the bird's skin, feathers or cloaca/intestine, which suggests some exchange of bacteria between the incubating bird and the eggshell. Furthermore, incubation appeared to promote the abundance of antibiotic producing taxa on the eggshell, which may hypothetically inhibit growth of many bacteria including pathogenic ones. Our results suggest that the future studies should focus on simultaneous monitoring of absolute abundance as well as relative abundance in communities on eggshells, nest materials and the incubating bird's body.

\section{Introduction}

Since microbial communities on eggshells may affect hatching success and nestling's physiological condition [1-3], the role of incubation behavior has been recently expanded from the classical function of controlling the thermal conditions of embryogenesis to manipulating the microbiome communities on eggshells [4-8]. Cook et al. (2015) [9] used a culture-based approach to study the eggshell microbial communities of a tropical wild bird species, Margarops fuscatus, and found a decrease in the percentage of eggs infected with pathogenic bacteria after incubation. Brandl et al. (2014) [6] studied bacterial communities of Reed Warbler's eggs and nestlings and found complete removal of haemolytic bacteria after incubation. Using high throughput sequencing, Lee et al. (2014) [8] studied microbial community structure of eggshells of the Oriental Magpie Pica serica and found a decrease in the relative abundance of pathogenic bacterial genera (Pseudomonas) and increase in harmless bacterial genera (Bacillus) after incubation.

On the other side, there were efforts made to characterize potential source of eggshell microbiome since eggs are known to be internally sterile at the time when they are laid $[9,10]$. Using culture-based methods, Ruiz-De-Castañeda et al. (2011) [11] found a positive correlation between bacterial loads of maternal cloaca and eggshells in the broods of the Pied Flycatcher (Ficedula hypoleuca). Using ARISA fingerprinting, Martínez-García et al. (2016) [12] demonstrated an association between nest materials' and eggshells' microbial communities of Hoopoes (Upupa epops). More recently, van Veelen et al. (2018) 
[10] studied Woodlarks (Lullula arborea) and Skylarks (Alauda arvensis) and found brood patch skin, feather, and nest material being the most important source of the microbiome of freshly laid eggs rather than maternal cloaca.

Nest materials, especially from the nest cup (in which the eggs are located), have often been assumed as a potential source of eggshell microbiome because they are in direct physical contact with the eggshells throughout incubation period. However, the dynamics and phylogenetic structure of the microbial communities on nest materials and eggshells during the incubation period have not been analyzed. Since eggshell microbiome starts to develop when the eggs are laid, it is important to monitor the changes in eggshell microbiome alongside with nest material microbiomes as both may continuously interact. Here, we aimed to understand the dynamics of microbial communities on eggshells and nest materials as well as interactions between these communities during incubation of the Oriental tit Parus minor using a high throughput sequencing technique. Under the general hypothesis that eggshell microbiome and nest material microbiome interact with each other and go through interactive dynamics during incubation by female birds, we aimed to verify the following four predictions that stem from four specific hypotheses:

Prediction 1) Before the onset of incubation, the microbial communities formed on the nest material will be more diverse and will show stronger phylogenetic clustering than the microbial communities on the eggshells. We hypothesize (Hypothesis 1) that nest materials of the Oriental tit, which include an underlying layer of green moss with overlaying grasses, rootlets, and animal hair forming the nest cup, contain bacterial communities that have already been established on those materials before they have been brought into the nests. Those communities may be ecologically and taxonomically specific for each type of nest material and each nest's local habitat. We hypothesize that this would lead to the higher diversity and possibly also stronger phylogenetic clustering (where a group of closely related species shares a trait, or suite of traits, that allow them to persist on a given type of nest materials/in a given local habitat). On the other hand, we expect that a typical eggshell microbiome at the onset of incubation has low abundance and low diversity of bacteria that are mainly coming from one source: the egg laying female [9-11]

Prediction 2) The initial communities of microbes on the eggshells and on nest materials will become more similar to each other during the incubation period. As the nest materials and the eggshells maintain direct contact with each other during incubation and because female birds rotate their eggs from time to time during incubation, as well as move inside the nest touching eggs and nest materials, we hypothesize (Hypothesis 2) that the microbial communities of eggshells and nest materials will become more similar to each other near the end of incubation period.

Prediction 3)There will be higher proportion of beneficial bacteria and lower proportion of pathogenic bacteria after incubation in both of the communities, on the eggshells and on the nest materials. The consistent presence of an incubating bird promotes growth of beneficial bacteria on the eggshells [6, 8, 9]. We hypothesize (Hypothesis 3) that due to the direct contact between female's body and the eggshells and the nest-cup materials throughout incubation, and due to transition of microbes between eggs and 
nest materials, beneficial bacteria will be present in higher proportion on both, the eggshells and on the nest-cup materials after incubation.

Prediction 4) Eggshell microbial communities will experience greater phylogenetic turnover compared to nest material microbial communities during incubation. We hypothesize (Hypothesis 4) that a nest material community within a nest remains more stable during incubation and experience less phylogenetic turnover because it is already established on the surface of nest materials before they are transported to the nest, and because the main source of new taxa is the relatively less diverse (Prediction 1) eggshell community on eggs that rest on the nest materials. On the other hand, eggshell microbial communities are expected to experience more environmental changes during incubation as eggshell is in contact with the female bird who provides warmth, and by frequently leaving the nest for foraging the female may bring more microbes from the environment. Additionally, as the typical eggshell microbiome is expected to start with a low-abundance and low-diversity community, a rapid succession of communities may be expected (mirroring the classical ecological succession) during incubation period. All these processes may result in higher phylogenetic turnover in eggshell communities.

\section{Methods}

\section{Field sampling}

We used nest boxes located in the campus of Seoul National University, which is located in the middle of Mt. Gwanak (a.s.I 632 m; Table 1), South Korea. Mt. Gwanak harbors temperate forests with an annual average temperature of $9^{\circ} \mathrm{C}$ and an annual average relative humidity of $74 \%$ at the summit (https://data.kma.go.kr/cmmn/main.do, Korea Meteorological Administration, 2018). The breeding season of Oriental tits starts from late March until late July in Mt. Gwanak. In 2018, we collected samples of eggshell microbes and nest materials from nine breeding nests. Oriental tits lay 8-9 eggs on average and in most cases start incubation with the production of the last egg. We used sterilized cotton-tipped swabs dipped in sodium phosphate buffer solution $(\mathrm{pH} 7.2,0.2 \mathrm{M})$ to collect eggshell microbial samples. To obtain sufficient biomass of microbes, we swabbed the surface of three whole eggs before incubation started ("before incubation" samples) and a different set of three eggs near the end of incubation period ("after incubation" samples; $3-5$ days before expected hatching date or 12 days after the last egg was laid). Nest materials were collected from the nest cup right beneath the eggs on the day of the eggshell microbe sampling. Samples were transported to the lab in Seoul National University within 4 hrs after collection and stored at $-20^{\circ} \mathrm{C}$, awaiting DNA extraction.

\section{DNA extraction, PCR, and sequencing}

DNA was extracted from the eggshell and the nest material samples using the PowerSoil Kit (Qiagen, Carlsbad, CA) following manufacturer's instruction. Extracted DNAs were shipped to CGEB-IMR (Canada) for polymerase chain reaction (PCR) and high-throughput sequencing. PCR targeting bacterial V4-V5 region of 16S rRNA gene was performed using modified 515f (5'-GTGYCAGCMGCCGCGGTAA-3') and 
806r (5'- GGACTACNVGGGTWTCTAAT-3') primers described in Walters et al. (2016) [13]. Amplified DNAs were paired-end sequenced ( 2 × $300 \mathrm{bp})$ using the Illumina MiSeq platform.

\section{Sequence processing}

Raw fastq sequence files were uploaded to NCBI SRA (sequence read archive) under project ID of PRJNA629431. Paired-end sequence reads were joined together using PANDAseq v. 2. 8. (Masella et al 2012). Sequences were further processed following the MiSeq SOP (https://www.mothur.org/wiki/MiSeq_SOP) using Mothur v. 1. 42. 3 [14]. For sequence alignment and classification, Silva database v. 132 [15] was used. Operational taxonomic units (OTUs) were assigned based on $97 \%$ similarity using the OptiClust algorithm [16]. Archaeal, chloroplast, and eukaryotic sequences were removed. Sequences were subsampled into 3,123 reads for statistical analysis.

\section{Phylogenetic analysis on the community structure}

Maximum-likelihood phylogenetic tree was generated using FastTree v. 2. 1. 3 [17]. To evaluate phylogenetic clustering in the microbial community of each sample, we calculated the standardized effect size of the mean nearest taxon distance (SES.MNTD) $[18,19]$ using the "ses.mntd" function in R package "picante" [20] with 999 randomized null models. The MNTD represents the mean distance separating each species in the community from its closest relative and thus allows to evaluate phylogenetic aspects of the community structure. Standardized effect size of the mean nearest taxon distance (SES.MNTD) describes the difference between phylogenetic distances in the observed community compared to a null community generated by a randomization method (and divided by the standard deviation of phylogenetic distances in the null data [19] and negative values of SES.MNTD indicate closely related taxa co-occurred more often than predicted in the null model.

To assess the community changes (i.e. phylogenetic turnover) during incubation, we determined pairwise evolutionary distances between communities before and after incubation using $\beta$-nearest taxon index ( $\beta N T I)$ [19] in the Phylocom software [21]. The values of $\beta N T I$ within the range from -2 to +2 are interpreted as indicating phylogenetic turnover not significantly different from a null expectation [19], while values below this range indicate significantly lower phylogenetic turnover during incubation than expected by chance.

\section{Statistical analysis}

To compare beta diversity and SES.MNTD of the samples before and after incubation, paired Wilcoxon signed rank test was used. As each variable represents a different specific hypothesis and we were interested in specific sets of comparisons (thus 'planned' comparisons, e.g. before vs after incubation, eggshell vs nest materials), we did not to use correction of the significance level for multiple comparisons (e.g. Bonferroni correction, Holm-Bonferroni method [22]). To compare OTU richness and Shannon diversity between the four different types of samples (eggshell before incubation, eggshell after incubation, nest material before incubation, nest material after incubation), we used linear mixed effect 
models and Tukey's posthoc test with nest ID included as a random variable. Bray-Curtis dissimilarity between samples were calculated based on square root transformed OTU table and visualized through non-metric multidimensional scaling (nMDS) plots using the Primer 6 software [23]. Analysis of similarity (ANOSIM) test was performed using the Primer 6 software [23] with 999 permutations. To test if there is any difference in community composition at phylum level and genus level after incubation, differential abundance analysis $[24,25]$ were performed using the "DESeq2" package in $\mathrm{R}$ \{Love, 2014 \#81\}. Pseudocount of 1 was applied for each the samples at both phylum level and genus level to calculate log2-fold change during incubation. p-values were adjusted for differential abundance analysis using the method described by Benjamini and Hochberg (1995) [26].

\section{Results}

\section{Changes in microbiome composition during incubation}

Eggshell microbial communities were distinct from nest material microbial communities both before and after incubation (Fig. 1, Table S1). There was a significant difference in the composition of eggshell microbial communities before and after incubation (Table S1). In contrast, no significant difference was found in the nest material microbial communities before and after incubation (Table S1). Bray-Curtis dissimilarities of the microbial communities before and after incubation were higher in eggshells compared to nest materials (Fig. 2-a). The eggshell microbial communities were similarly distinct from the nest material microbial communities before and after incubation as indicated by no significant difference in their dissimilarities (Fig. 2-b).

The most abundant phylum found on eggshells was Proteobacteria, followed by Firmicutes, Actinobacteria, Bacteroidetes, and Planctomycetes (Fig. 3). The major phylum composition was similar in nest material microbial community, where Proteobacteria was the most abundant phylum, followed by Bacteroidetes, Firmicutes, Actinobacteria, and Acidobacteria. Differential abundance analysis (Table S2) shows that Proteobacteria became significantly less abundant after incubation on the eggshells. On the other hand, Firmicutes and Actinobacteria became significantly more abundant after incubation on the eggshells. There was no significant change in the abundance of bacterial phyla in nest materials.

The most abundant genus found on eggshells was Psychrobacter, followed by Streptomyces, Rhodococcus, Shpingobacterium, and Pedobacter (Fig. 4). On the other hand, the most abundant genus found in nest materials was Massilia, followed by Pedobacter, Flavobacterium, Chryseobacterium, Acinetobacter, and Shpingobacterium. Differential abundance analysis (Table S3) shows significant decrease in the abundance of Psychrobacterduring incubation in the eggshell microbiome. In contrast, Paenibacillus became more abundant after incubation on the eggshell.

\section{Change in microbial diversity during incubation}

OTU richness and Shannon diversity were lower in the eggshell microbial communities compared to nest material microbial communities both before and after incubation (Fig. 5, Table S4-5). There was no 
significant change in OTU richness and Shannon diversity during incubation both on the eggshells and in nest materials (Fig. 5, Table S4-5).

\section{Change in the phylogenetic community structure during incubation}

In all of the samples, closely related taxa co-occurred more often than predicted in the null model (SES.MNTD < 0) (Table S6). The SES.MNTD value was significantly lower in the nest material microbial communities than in the eggshell microbial communities both before (paired Wilcoxon signed rank test, $W=0, p=0.004$ ) and after (paired Wilcoxon signed rank test, $W=0, p=0.004$ ) incubation (Fig. 6). This indicates that eggshell communities comprised taxa that were phylogenetically more distant from each other than were taxa in the nest material communities.

$\beta N T I$ values for the nest microbial communities were overall lower than -2 indicating that their phylogenetic structure remained significantly more stable during incubation that expected by chance (Fig. 7). However, $\beta N T I$ of the eggshell microbial communities ranged from -2 to +2 , indicating no significant differences in phylogenetic turnover compared to what is be expected by chance, and suggesting that processes other than phylogenetic relationship among taxa were responsible for shaping the eggshell communities during incubation.

\section{Discussion}

\section{Composition of microbial communities}

In this study, we have examined the bacterial communities on eggshells and nest materials. The composition of microbial community on the eggshell was significantly different that on the nest material at OTU level, although the major phylum composition was rather similar. The most abundant phylum found in this study was Proteobacteria, which is one of the dominant phyla found in soil [27], phyllosphere [28], potential nest material sources such as moss crusts [29], and also on avian eggshells $[7,8,10,30,31]$.

Fimicutes, which was significantly more abundant in eggshell samples collected after incubation compared to the ones collected before incubation, is often found in animal's gut microbiota including that of wild bird species [32]. Considering the lower abundance of Firmicutes in nest materials compared to eggshells, it is possible that the species belonging to Fimicutes, which might have originated from parent bird's gastrointestinal tract, are competitively dominant over the other taxa on eggshells probably because the temperature on the eggshell is closer to that in gastrointestinal tract (due to incubation). Lee et al. (2014) [8] also found overall higher averaged relative abundance of Firmicutes in incubated eggs and found Bacillus (belonging to Firmicutes), which includes species producing antibiotics [33], being significantly more abundant after incubation. It is however also possible that there is a consistent load of Firmicutes from external sources other than nest materials during the incubation. 
The most abundant genus in the eggshell microbiome was Psychrobacter and they were more abundant on the eggshell before incubation and their relative abundance decreased significantly with incubation. As its name suggests, they are mostly found in cold environments such as Arctic glacier or Antarctic [3436]. However, they have also been found in other eggshell studies. Wang et al. (2011) [3] studied Western Bluebird, Tree Swallow, and Violet-green Swallow's eggs in California and isolated Psychrobacter species from their samples. Grizard et al. (2014) [7] studied homing pigeons in Netherlands and found an OTU that has $99 \%$ sequence similarity with Psychrobacter glacinola. Although it has not been discussed in a detail in these studies, it seems Psychrobacter sp. can also be found in temperate environments. Further studies on the sources of Psychrobacter in the eggshells and their survival strategies in an ambient temperature would be interesting. Decrease in the relative abundance of Psychrobacter on eggshells during incubation might be due to increase in temperature both outside and inside of nests.

Massilia, which was found to be the most abundant genus in the nest material microbiome, has been isolated from a variety of environments, encompassing soil, atmosphere, freshwater, phyllosphere, rizosphere, and in blood samples [6,37]. They have also been found in hen's flea (Ceratophyllus gallinae) samples in Great tit's nests \{Aivelo, 2020 \#55\}. The presence of plants and rootlets in the nest may be related to the abundance of Massilla.

Goodenough and Stallwood (2010) \{Goodenough, 2010 \#11\} accessed bacterial and fungal microbial composition of blue tit and great tit nest material using culture-based method and found species belonging to Bacillus, Pseudomonas, Enterobacter, and Staphylococcus dominating. Although some of those genera were also detected in our study, their relative abundance was much lower than it was found in Goodenough and Stallwood (2010) [38]. This could be due to the different methods used in the two studies. Animal pathogens have been studied very well and have been cultured for more than decades. In contrast, most of the bacterial taxa found in environmental samples are uncultured or recently culture lacking detailed information. The result from our study suggests that there are much more diverse bacterial groups present in bird's nest and it is not necessarily dominated by pathogenic taxa.

Another finding in our study is that there is a lack of incubation effect on bacterial diversity even though there is an effect on bacterial composition. Grizard et al. (2014) [7] found reduction in bacterial diversity after incubation on the eggshells of homing pigeon Columba livia. Lee et al. (2014) [8] found the same pattern on the eggshells of Oriental magpie, Pica serica. The different pattern found in our study could be due to the ecological difference between studied species, for example, breeding season and the use of different types of nest materials. Below we discuss the remaining results in the framework of testing of the four predictions (see Introduction).

\section{Testing the predictions}

Prediction 1) Before the onset of incubation, the microbial communities formed on the nest material will be more diverse and will show stronger phylogenetic clustering than the microbial communities on the eggshells. 
As expected, nest material microbial communities had higher diversity compared to eggshell microbial communities. Also, the co-occurrence of closely related taxa (which have high possibility to share genes with similar functions) was stronger in the nest material microbiome (lower SES.MNTD). These results support the idea that the nest materials contain already established microbiome whereas the eggshell microbiome did not have sufficient time to be established. However, greater diversity in nest material microbial communities than that on eggshells remained similar even after incubation. This suggests that, even with direct contact, eggshell microbiome experiences independent change from nest materials (see below).

Prediction 2) The initial communities of microbes on the eggshells and on nest materials will become more similar to each other during the incubation period.

We found that the eggshell microbial community does not necessarily become more similar to nest material microbial community after incubation. In fact, the changes in nest material microbial communities during incubation were minimal compared to those of the eggshell microbial communities. The nest materials might have had less chance to be affected by incubation, because they are placed under the eggs and do not interact directly with the female's brood patch. Another possibility is that the nest materials, as they are not in direct contact with the female's body, they are maintained at lower temperature than eggshells throughout the incubation period, which may differentially limit the proliferation of certain bacteria. The huge changes in the eggshell microbiome during incubation may imply rapid successional processes occurring during incubation. It could be useful to collect another sets of samples in the middle of incubation to have a glance at the spectrum of the changes.

Prediction 3) There will be higher relative abundance of beneficial bacteria and lower relative abundance of pathogenic bacteria after incubation both in the eggshell and nest materials.

Overall, we found high variability between the samples in their taxonomic composition. However, we were still able to find Psychrobacter and Paenibacillus showing significant differences in their relative abundance after incubation.Psychrobacter are rare opportunistic human pathogens [39] and their pathogenicity against birds has not been reported. Their relationship with birds has been described only in a limited number of studies, so it is difficult to conclude if they are beneficial or harmful to birds in general.

The relative abundance of Paenibacillus was higher in the eggshell samples collected after incubation than the ones collected before incubation. Many of Paenibacillus species are known to produce antibiotics $[40,41]$. Their ability to produce antibiotics might be related to prevention of pathogenic bacterial growth and it may contribute to overall change in the composition of bacterial communities of eggshells and nest materials. Some of the species belonging to Paenibacillus are known to have keratinolytic activity, potentially contributing to bird feather degradation [42]. The increase in Paenibacillus after incubation might be originated from the feather of female birds through continuous contact with their eggs and could possibly affect chick's feather development. 
In conclusion, there is no evidence that potentially pathogenic taxa decreased their relative abundance. However, as we did not measure the absolute abundance, we cannot exclude the possibility that indeed the abundance of potentially pathogenic bacteria decreased assuming that that general abundance of bacteria might have decreased like in some previous studies $[1,4,9]$. We have evidence suggesting that the proportion of antibiotic-producing, hence potentially beneficial for birds, bacteria increased in the eggshell communities during incubation.

Prediction 4) Eggshell microbial community will experience greater phylogenetic turnover compared to nest material microbial community during incubation.

As expected, the phylogenetic turnover in nest material microbial communities were minimal. Similarly, with what we mentioned in Prediction 1, this result might be due to their relative "age", as they have been already developed and established long before the nest materials are transported to the nest during nest building stage. On the other hand, eggshell microbial community showed similar level of phylogenetic turnover from what can be expected by chance, implying relatively new microbial communities were developing during incubation. This implies that the phylogenetic turnover occurring in eggshell microbial community is not related with (or influenced by) the dynamics in nest material microbial community. More likely is that the microbes originating from parents shape the dynamics in eggshell microbial community during incubation $[10,11,43,44]$. For instance, we found noticeable increase in the proportion of Firmicutes on the eggshell and Firmicutes include species of microbes present in uropygial gland [45] gastrointestinal tract $[38,46,47]$, or feathers [48]. It is plausible that the microbes originating from parents, either through contact with cloaca (when laying) or with uropygial gland and feathers (during incubation), are transferred once or continuously to the eggshell and shape the dynamics and phylogenetic turnover in eggshell microbial community.

\section{Conclusions}

In summary, we found significant changes in the structure of microbial communities on eggshells during incubation but not in communities on nest materials. The result from the phylogenetic analysis supports the idea that the minimal changes in nest materials could be due to their relatively long successional history. In contrast to the findings from other studies, we found no evidence of a decrease in the relative abundance of taxa containing pathogenic species, which could be due to the difference in the life history traits of the studied bird species [49]. However, the increase in relative abundance of taxa producing antibiotics may be viewed as hypothetically adaptive direction of change of bacterial communities on eggshells because antibiotics may inhibit growth of pathogens. This study demonstrates for the first time the changes in microbial community structure in the nest materials microbiome during incubation. As there has been no study which characterized microbial composition of the Oriental tit eggs and nest materials using high throughput sequencing, the results from this study add knowledge for the future comparative analyses across different avian taxa.

\section{Declarations}




\section{Funding}

This work was supported by National Research Foundation of Korea (NRF) via grant 3344-20180017, DGIST Start-up Fund Program of the Ministry of Science and ICT via grant 2021010026, and by the BK Korea 21 (Grant number: 5253-20180100/21A20131212006).

\section{Conflicts of Interest}

The authors declare no conflict of interest.

\section{Availability of data and material}

Raw fastq sequence files were uploaded to NCBI SRA (sequence read archive) under project ID of PRJNA629431.

\section{Code availability}

Not applicable

\section{Authors' contributions}

Hokyung Song: Conceptualization, Investigation, Formal analysis, Writing - original draft, Visualization, Funding acquisition. Keesan Lee, Injae Hwang, Eunjeong Yang, Jungmoon Ha, and Woojoo Kim: Investigation (nest monitoring and sample collection). Sungjin Park: Investigation (nest monitoring), Writing - review \& editing. Hyunjoon Cho: Formal analysis. Sang-im Lee and Piotr Jablonski: Conceptualization, Investigation, Writing - review \& editing, Funding acquisition.

\section{Ethics approval}

This research was conducted following national and international guidelines. The methods used in this study were reviewed and approved by Institutional Animal Care and Use Committee of Seoul National University (No. SNU-180727-1).

\section{Consent to participate}

Not applicable

\section{Consent for publication}

Not applicable

\section{References}

1. Cook MI, Beissinger SR, Toranzos GA, Rodriguez RA, Arendt WJ (2003) Trans-shell infection by pathogenic micro-organisms reduces the shelf life of non-incubated bird's eggs: a constraint on the 
onset of incubation? Proceedings of the Royal Society of London Series B: Biological Sciences 270: 2233-2240. doi: 10.1098/rspb.2003.2508

2. Peralta-Sánchez JM, Martínez-Bueno M, Martín-Vivaldi M, Soler JJ (2010) Nest Bacterial Environment Predict Hatching Success of Wild European Birds. A Comparative Study. University of granada

3. Wang JM, Firestone MK, Beissinger SR (2011) Microbial and environmental effects on avian egg viability: Do tropical mechanisms act in a temperate environment? Ecology 92:1137-1145. doi:10.1890/10-0986.1

4. Shawkey MD, Firestone MK, Brodie EL, Beissinger SR (2009) Avian incubation inhibits growth and diversification of bacterial assemblages on eggs. PloS one 4:e4522-e4522. doi:10.1371/journal.pone.0004522

5. Ruiz-De-Castañeda R, Vela Al, González-Braojos S, Briones V, Moreno J (2011) Drying eggs to inhibit bacteria: Incubation during laying in a cavity nesting passerine. Behavioural Processes 88: 142-148. doi: https://doi.org/10.1016/j.beproc.2011.08.012

6. Brandl HB, van Dongen WFD, Darolová A, Krištofík J, Majtan J, Hoi H (2014) Composition of Bacterial Assemblages in Different Components of Reed Warbler Nests and a Possible Role of Egg Incubation in Pathogen Regulation. PLOS ONE 9:e114861. doi:10.1371/journal.pone.0114861

7. Grizard S, Dini-Andreote F, Tieleman BI, Salles JF (2014) Dynamics of bacterial and fungal communities associated with eggshells during incubation. Ecology Evolution 4:1140-1157. doi:10.1002/ece3.1011

8. Lee WY, Kim M, Jablonski PG, Choe JC, Lee S-i (2014) Effect of Incubation on Bacterial Communities of Eggshells in a Temperate Bird, the Eurasian Magpie (Pica pica). PLOS ONE 9:e103959. doi:10.1371/journal.pone.0103959

9. Cook MI, Beissinger SR, Toranzos GA, Arendt WJ (2005) Incubation reduces microbial growth on eggshells and the opportunity for trans-shell infection. Ecol Lett 8:532-537. doi:10.1111/j.14610248.2005.00748.x

10. van Veelen HPJ, Salles JF, Tieleman BI (2018) Microbiome assembly of avian eggshells and their potential as transgenerational carriers of maternal microbiota. The ISME Journal 12:1375-1388. doi:10.1038/s41396-018-0067-3

11. Ruiz-De-Castañeda R, Vela Al, Lobato E, Briones V, Moreno J (2011) Bacterial Loads on Eggshells of the Pied Flycatcher: Environmental and Maternal Factors. The Condor 113:200-208. doi:10.1525/cond.2011.100035

12. Martínez-García Á, Martín-Vivaldi M, Rodríguez-Ruano SM, Peralta-Sánchez JM, Valdivia E, Soler JJ (2016) Nest Bacterial Environment Affects Microbiome of Hoopoe Eggshells, but Not That of the Uropygial Secretion. PloS one 11:e0158158-e0158158. doi:10.1371/journal.pone.0158158

13. Walters W, Hyde ER, Berg-Lyons D, Ackermann G, Humphrey G, Parada A, Gilbert JA, Jansson JK, Caporaso JG, Fuhrman JA, Apprill A, Knight R (2016) Improved Bacterial 16S rRNA Gene (V4 and V4- 
5) and Fungal Internal Transcribed Spacer Marker Gene Primers for Microbial Community Surveys. mSystems 1:e00009-e00015. doi:10.1128/mSystems.00009-15

14. Schloss PD, Westcott SL, Ryabin T, Hall JR, Hartmann M, Hollister EB, Lesniewski RA, Oakley BB, Parks DH, Robinson CJ, Sahl JW, Stres B, Thallinger GG, Van Horn DJ, Weber CF (2009) Introducing mothur: Open-Source, Platform-Independent, Community-Supported Software for Describing and Comparing Microbial Communities. Appl Environ Microbiol 75:7537-7541. doi:10.1128/aem.0154109

15. Quast C, Pruesse E, Yilmaz P, Gerken J, Schweer T, Yarza P, Peplies J, Glöckner FO (2012) The SILVA ribosomal RNA gene database project: improved data processing and web-based tools. Nucleic Acids Res 41:D590-D596. doi:10.1093/nar/gks1219

16. Westcott SL, Schloss PD (2017) OptiClust, an Improved Method for Assigning Amplicon-Based Sequence Data to Operational Taxonomic Units. mSphere 2:e00073-e00017. doi:10.1128/mSphereDirect.00073-17

17. Price MN, Dehal PS, Arkin AP (2010) FastTree 2 - Approximately Maximum-Likelihood Trees for Large Alignments. PLOS ONE 5:e9490. doi:10.1371/journal.pone.0009490

18. Webb CO, Ackerly DD, McPeek MA, Donoghue MJ (2002) Phylogenies and Community Ecology. Annu Rev Ecol Syst 33:475-505. doi:10.1146/annurev.ecolsys.33.010802.150448

19. Stegen JC, Lin X, Konopka AE, Fredrickson JK (2012) Stochastic and deterministic assembly processes in subsurface microbial communities. The ISME Journal 6:1653-1664. doi:10.1038/ismej.2012.22

20. Kembel SW, Kembel MSW (2019) Package 'picante\&\#8217

21. Webb CO, Ackerly DD, Kembel SW (2008) Phylocom: software for the analysis of phylogenetic community structure and trait evolution. Bioinformatics 24

22. Holm S (1979) A Simple Sequentially Rejective Multiple Test Procedure. Scand J Stat 6:65-70

23. Clarke KR, Clarke K, Gorley K, Clarke K, Gorley R (2006) PRIMER v6: user manual/tutorial

24. Paulson JN, Stine OC, Bravo HC, Pop M (2013) Differential abundance analysis for microbial markergene surveys. Nat Methods 10:1200-1202. doi:10.1038/nmeth.2658

25. Weiss S, Xu ZZ, Peddada S, Amir A, Bittinger K, Gonzalez A, Lozupone C, Zaneveld JR, VázquezBaeza Y, Birmingham A, Hyde ER, Knight R (2017) Normalization and microbial differential abundance strategies depend upon data characteristics. Microbiome 5:27. doi:10.1186/s40168-0170237-y

26. Benjamini Y, Hochberg Y (1995) Controlling the False Discovery Rate: A Practical and Powerful Approach to Multiple Testing. Journal of the Royal Statistical Society Series B (Methodological) 57:289-300

27. Janssen PH (2006) Identifying the Dominant Soil Bacterial Taxa in Libraries of $16 \mathrm{~S}$ rRNA and $16 \mathrm{~S}$ rRNA Genes. Appl Environ Microbiol 72:1719-1728. doi:10.1128/aem.72.3.1719-1728.2006 
28. Vorholt JA (2012) Microbial life in the phyllosphere. Nat Rev Microbiol 10:828-840. doi:10.1038/nrmicro2910

29. Navarro-Noya YE, Jiménez-Aguilar A, Valenzuela-Encinas C, Alcántara-Hernández RJ, Ruíz-Valdiviezo VM, Ponce-Mendoza A, Luna-Guido M, Marsch R, Dendooven L (2014) Bacterial communities in soil under moss and lichen-moss crusts. Geomicrobiol J 31:152-160

30. Grizard S, Versteegh MA, Ndithia HK, Salles JF, Tieleman BI (2015) Shifts in Bacterial Communities of Eggshells and Antimicrobial Activities in Eggs during Incubation in a Ground-Nesting Passerine. PLOS ONE 10:e0121716. doi:10.1371/journal.pone.0121716

31. Trudeau S, Thibodeau A, Côté J-C, Gaucher M-L, Fravalo P (2020) Contribution of the Broiler Breeders' Fecal Microbiota to the Establishment of the Eggshell Microbiota. Front Microbiol 11. doi:10.3389/fmicb.2020.00666

32. Grond K, Sandercock BK, Jumpponen A, Zeglin LH (2018) The avian gut microbiota: community, physiology and function in wild birds. J Avian Biol 49:e01788

33. Mannanov RN, Sattarova RK (2001) Antibiotics Produced by Bacillus Bacteria. Chem Nat Compd 37:117-123. doi:10.1023/A:1012314516354

34. Bowman JP, Cavanagh J, Austin JJ, Sanderson K (1996) Novel Psychrobacter species from Antarctic ornithogenic soils. Int J Syst Evol MicroBiol 46:841-848

35. Bozal N, Montes MJ, Tudela E, Guinea J (2003) Characterization of several Psychrobacter strains isolated from Antarctic environments and description of Psychrobacter luti sp. nov. and Psychrobacter fozii sp. nov. J Med Microbiol 53:1093-1100

36. Zeng Y-X, Yu Y, Liu Y, Li H-R (2016) Psychrobacter glaciei sp. nov., isolated from the ice core of an Arctic glacier. Int J Syst Evol Microbiol 66:1792-1798

37. Ofek M, Hadar Y, Minz D (2012) Ecology of Root Colonizing Massilia (Oxalobacteraceae). PLOS ONE 7:e40117. doi:10.1371/journal.pone.0040117

38. Goodenough AE, Stallwood B (2010) Intraspecific Variation and Interspecific Differences in the Bacterial and Fungal Assemblages of Blue Tit (Cyanistes caeruleus) and Great Tit (Parus major) Nests. Microb Ecol 59:221-232. doi:10.1007/s00248-009-9591-z

39. Joanna María O-A, José Miguel S-C, Fabiola G-A, Elizabeth G-D, Araceli R-C, Patricia A-P, Claudia W-A, Maribel G-V, Gloria L-Á, Adda Jeanette G-C, José Alberto D-Q, Alfonso M-T, José Ernesto R-G (2016) Fatal Psychrobacter sp. infection in a pediatric patient with meningitis identified by metagenomic next-generation sequencing in cerebrospinal fluid. Archives of microbiology 198:129-135. doi:10.1007/s00203-015-1168-2

40. Watve MG, Tickoo R, Jog MM, Bhole BD (2001) How many antibiotics are produced by the genus Streptomyces? Archives of microbiology 176:386-390. doi:10.1007/s002030100345

41. Grady EN, MacDonald J, Liu L, Richman A, Yuan Z-C (2016) Current knowledge and perspectives of Paenibacillus: a review. Microb Cell Fact 15:203. doi:10.1186/s12934-016-0603-7

42. Saarela M, Berlin M, Nygren H, Lahtinen P, Honkapää K, Lantto R, Maukonen J (2017) Characterization of feather-degrading bacterial populations from birds' nests - Potential strains for 
biomass production for animal feed. International Biodeterioration Biodegradation 123:262-268. doi:https://doi.org/10.1016/j.ibiod.2017.07.006

43. Engel K, Sauer J, Jünemann S, Winkler A, Wibberg D, Kalinowski J, Tauch A, Caspers BA (2018) Individual- and Species-Specific Skin Microbiomes in Three Different Estrildid Finch Species Revealed by 16S Amplicon Sequencing. Microb Ecol 76:518-529. doi:10.1007/s00248-017-1130-8

44. Ross AA, Rodrigues Hoffmann A, Neufeld JD (2019) The skin microbiome of vertebrates. Microbiome 7:79. doi:10.1186/s40168-019-0694-6

45. Bodawatta KH, Schierbech SK, Petersen NR, Sam K, Bos N, Jønsson KA, Poulsen M (2020) Great Tit (Parus major) Uropygial Gland Microbiomes and Their Potential Defensive Roles. Frontiers in Microbiology 11. doi: 10.3389/fmicb.2020.01735

46. Kropáčková L, Pechmanová H, Vinkler M, Svobodová J, Velová H, Těšičký M, Martin J-F, Kreisinger J (2017) Variation between the oral and faecal microbiota in a free-living passerine bird, the great tit (Parus major). PLOS ONE 12:e0179945. doi:10.1371/journal.pone.0179945

47. Fu R, Xiang X, Dong Y, Cheng L, Zhou L (2020) Comparing the intestinal bacterial communies of sympatric wintering Hooded Crane (Grus monacha) and Domestic Goose (Anser anser domesticus). Avian Research 11:13. doi:10.1186/s40657-020-00195-9

48. Burtt EH Jr, Ichida JM (1999) Occurrence of Feather-Degrading Bacilli in the Plumage of Birds. Auk 116:364-372. doi:10.2307/4089371

49. Peralta-Sánchez JM, Martín-Vivaldi M, Martín-Platero AM, Martínez-Bueno M, Oñate M, RuizRodríguez M, Soler JJ (2012) Avian life history traits influence eggshell bacterial loads: a comparative analysis. Ibis 154:725-737. doi:10.1111/j.1474-919X.2012.01256.X

\section{Tables}

Due to technical limitations, table 1 is only available as a download in the Supplemental Files section.

\section{Figures}




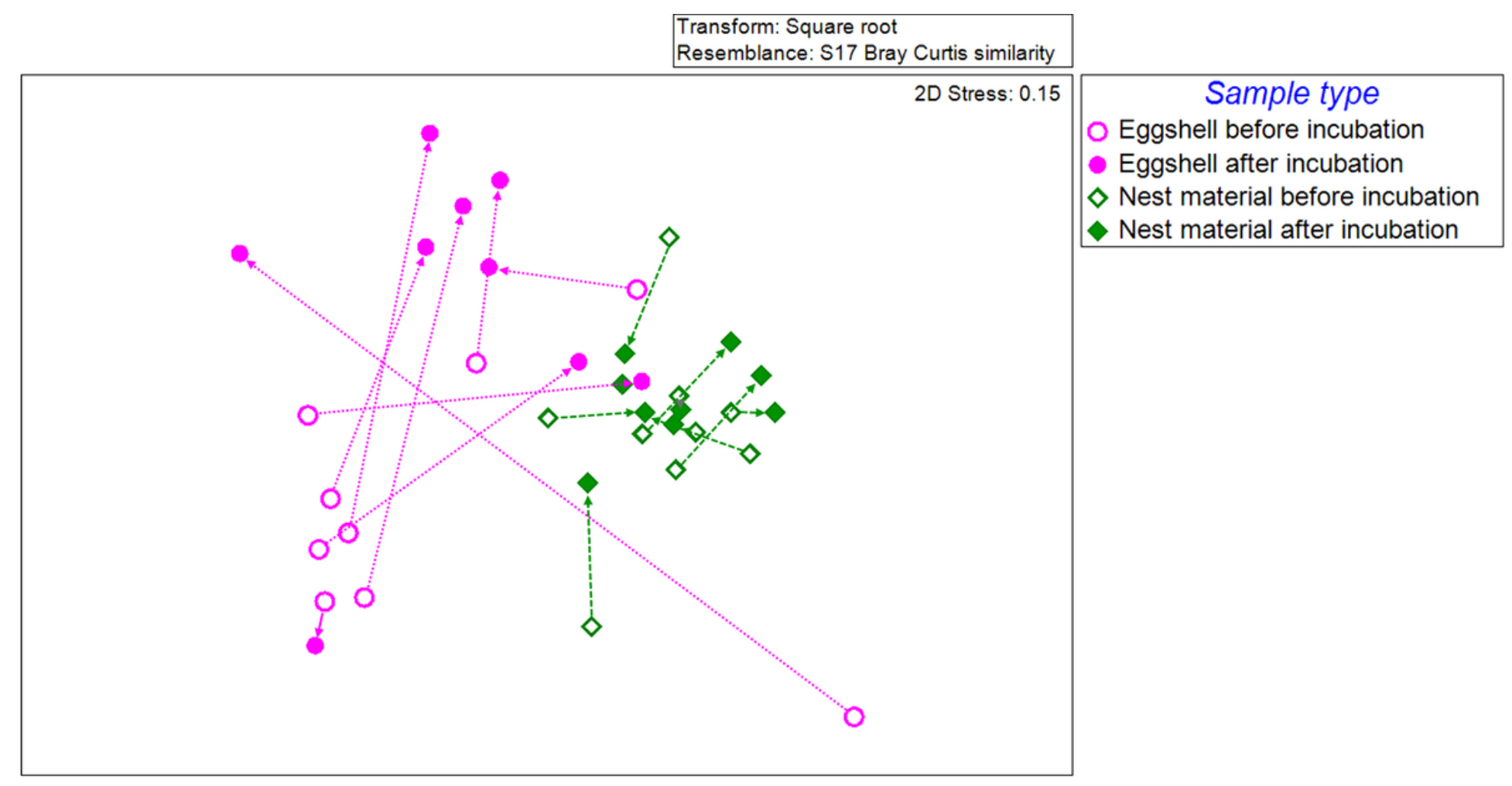

Figure 1

nMDS plot showing Bray-Curtis dissimilarities between samples.

(a)

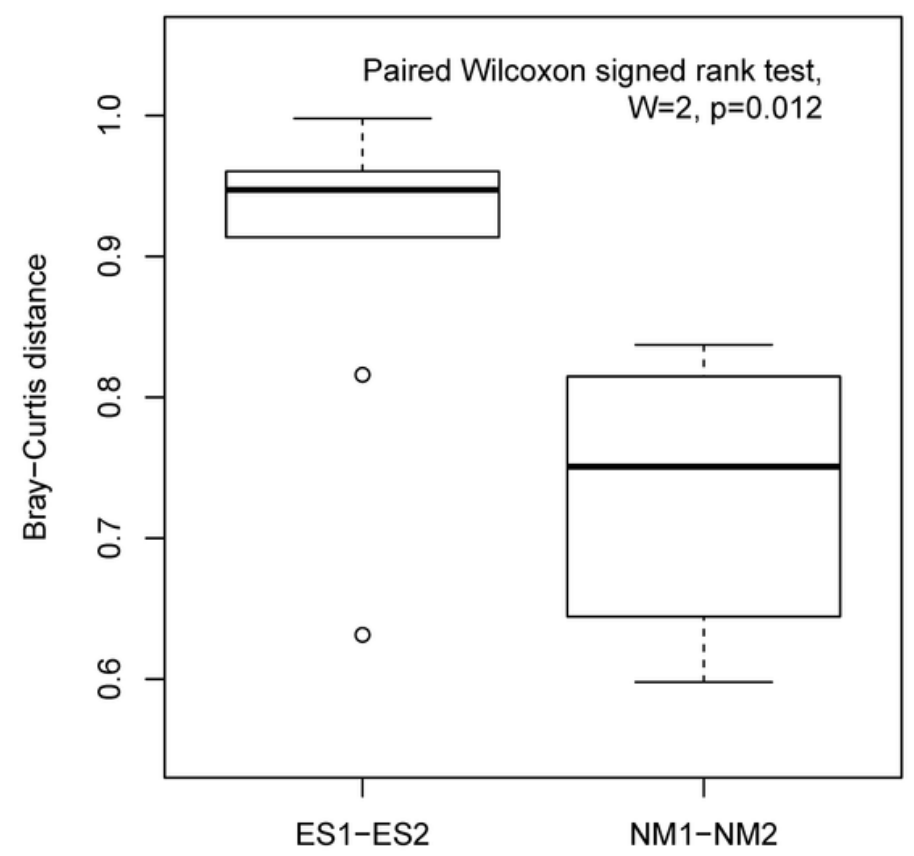

(b)

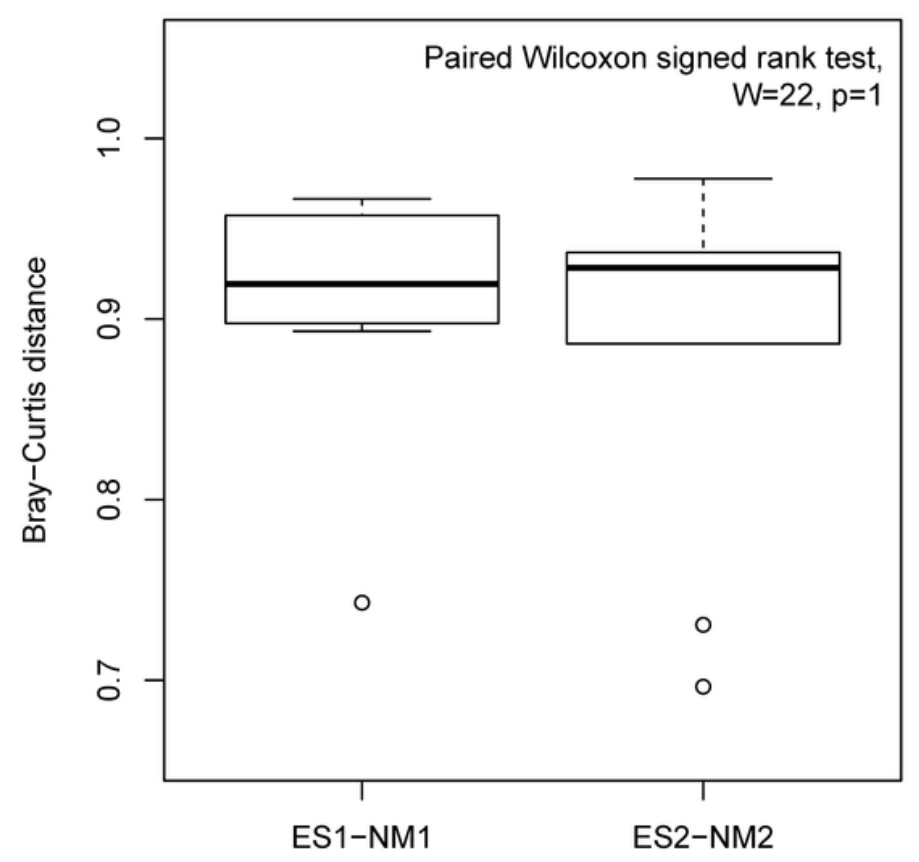

Figure 2 
Boxplots showing pairwise Bray-Curtis dissimilarities in the eggshell and nest material microbial communities. (a) Bray-Curtis dissimilarities of eggshell microbial communities before and after incubation (ES1 and ES2 respectively) compared with those of nest material microbial communities before and after incubation (NM1 and NM2 respectively). (b) Bray-Curtis dissimilarities of eggshell microbial communities and nest material microbial communities before incubation compared with those of eggshell and nest material microbial communities after incubation. Median: thick horizontal line; Upper and lower quartiles: upper and lower box edges; Minimum and maximum: the end of the lower and upper whiskers; Outliers: circles.

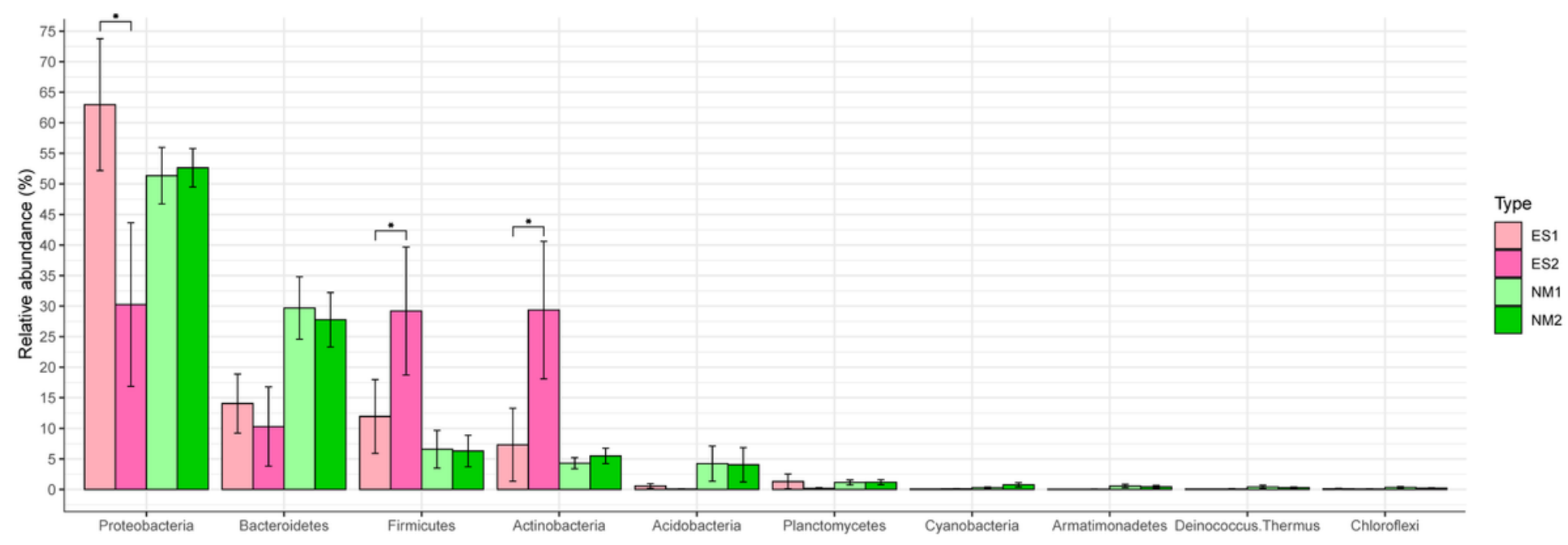

\section{Figure 3}

Relative abundance of the 10 most abundant bacterial phyla. Significant results (adjusted p-value $<0.05$ ) from the differential abundance analysis are shown as asterisks $\left(^{*}\right)$. ES1- eggshells before incubation; ES2 - eggshells near the end of incubation; NM1- nest materials before incubation; NM2 - nest material near the end of incubation.

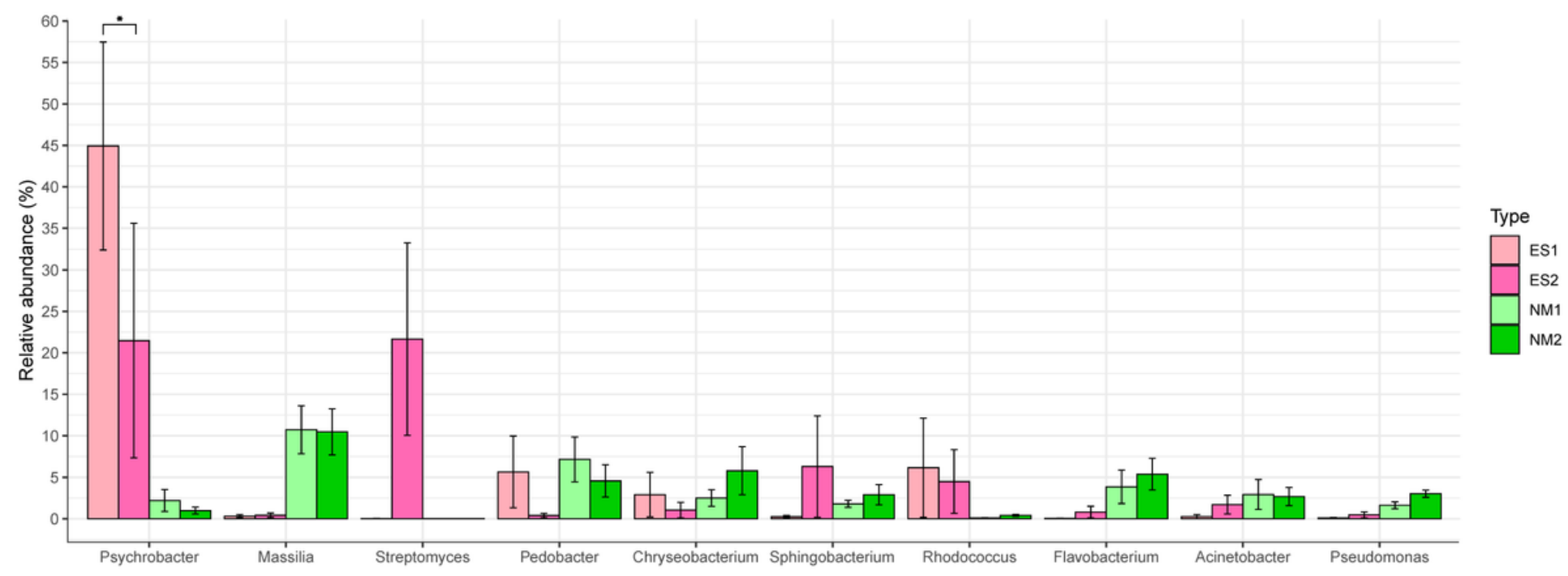

Figure 4 
Relative abundance of the 10 most abundant bacterial genera. Significant results (adjusted p-value $<0.05)$ from the differential abundance analysis are shown as asterisks $(*)$. ES1- eggshells before incubation; ES2 - eggshells near the end of incubation; NM1- nest materials before incubation; NM2 - nest material near the end of incubation.

(a)

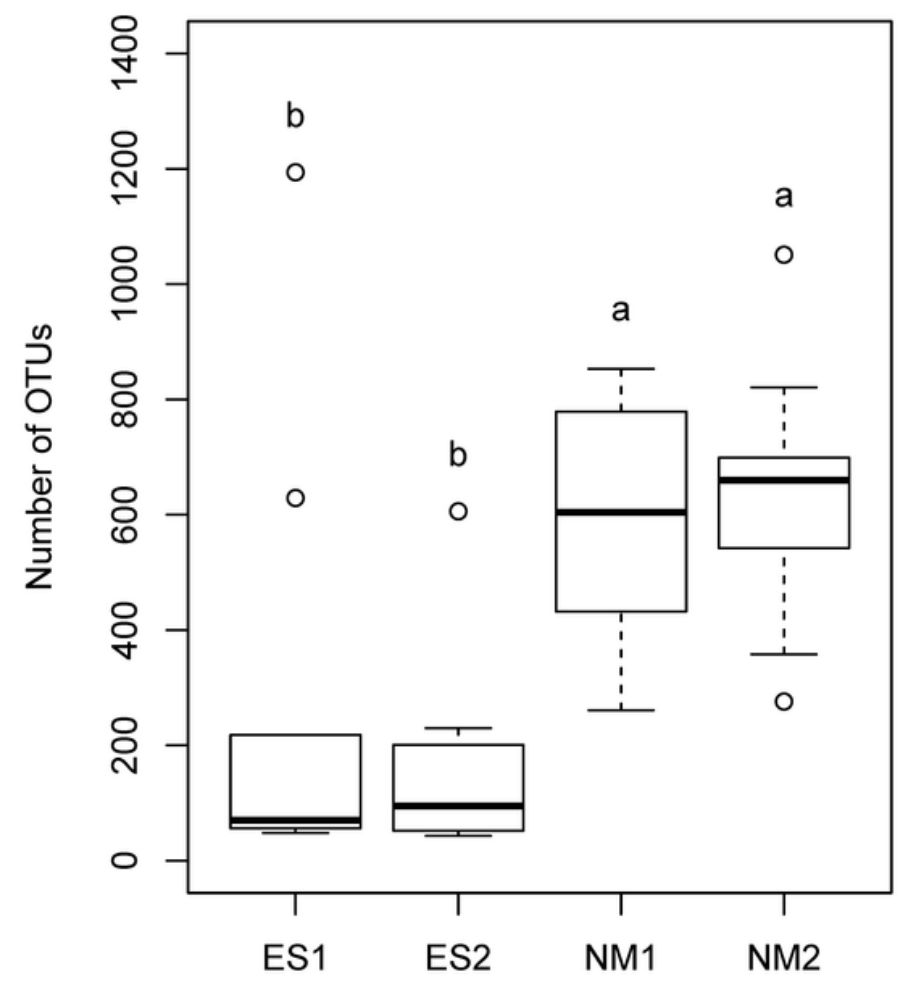

(b)

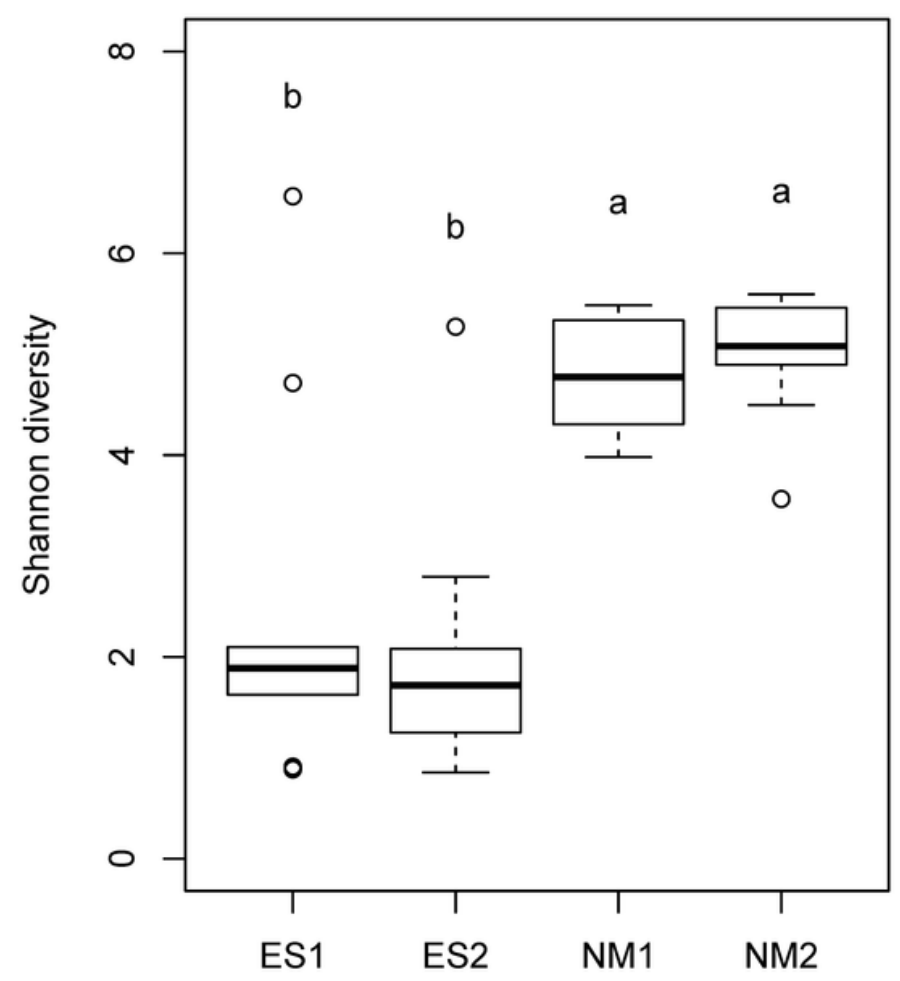

Figure 5

Effect of incubation on the diversity of microbiome on eggshells and nest materials. The number of OTUs (a) and Shannon diversity index (b) of bacterial communities for four types of samples: ES1- eggshells before incubation; ES2 - eggshells near the end of incubation; NM1- nest materials before incubation; NM2 - nest material near the end of incubation. Posthoc test results with $p<0.05$ after controlling for nest ID are shown as alphabet. 


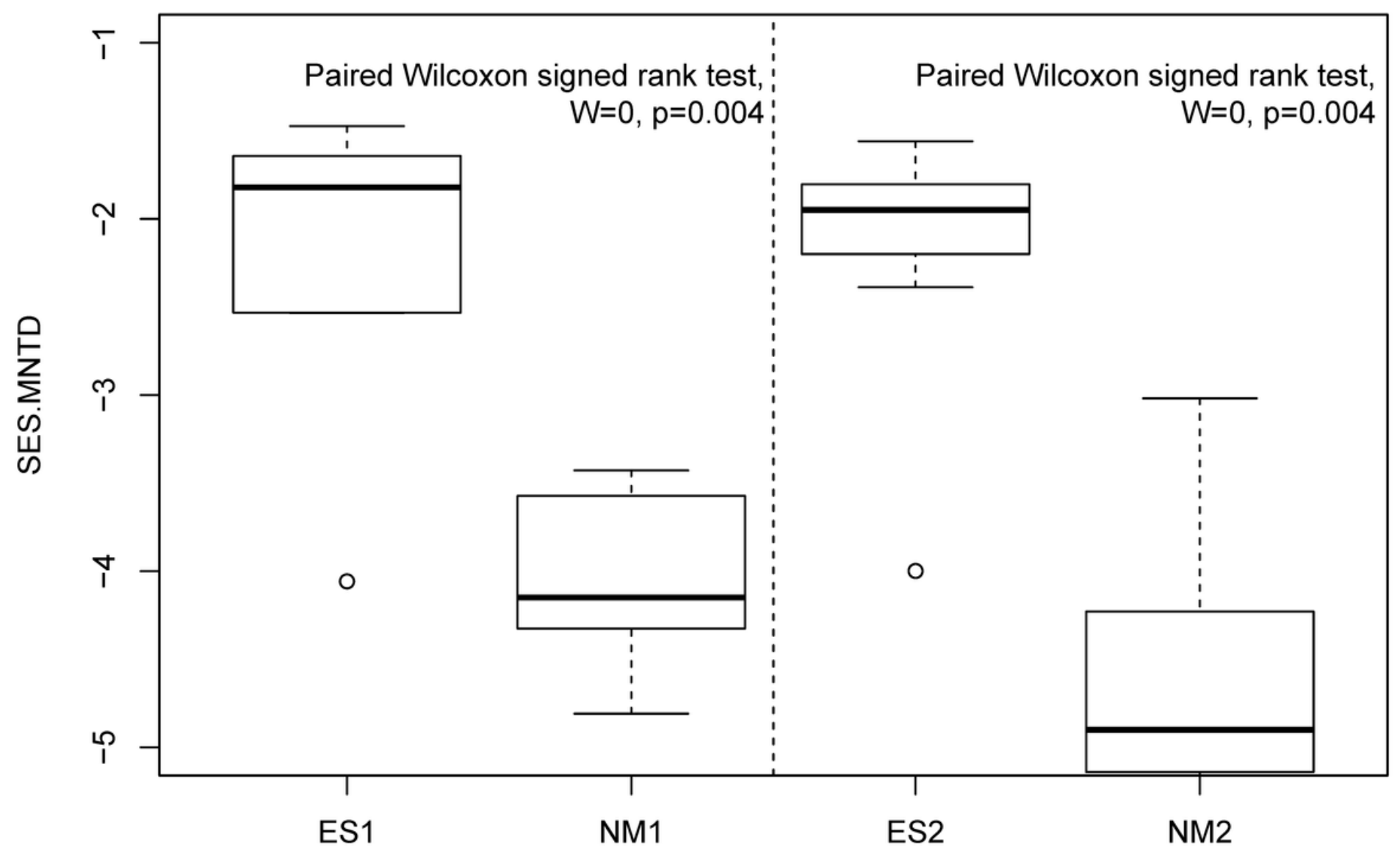

Figure 6

Comparison of standardized effect size of the mean nearest taxon distance (SES.MNTD) between eggshell (ES1, ES2) and nest material (NM1, NM2) microbiomes before incubation started (ES1, NM1) and near the end of incubation (ES2, NM2) in Oriental tit (Parus minor) nests ( $n=9$; Table 1$)$. 


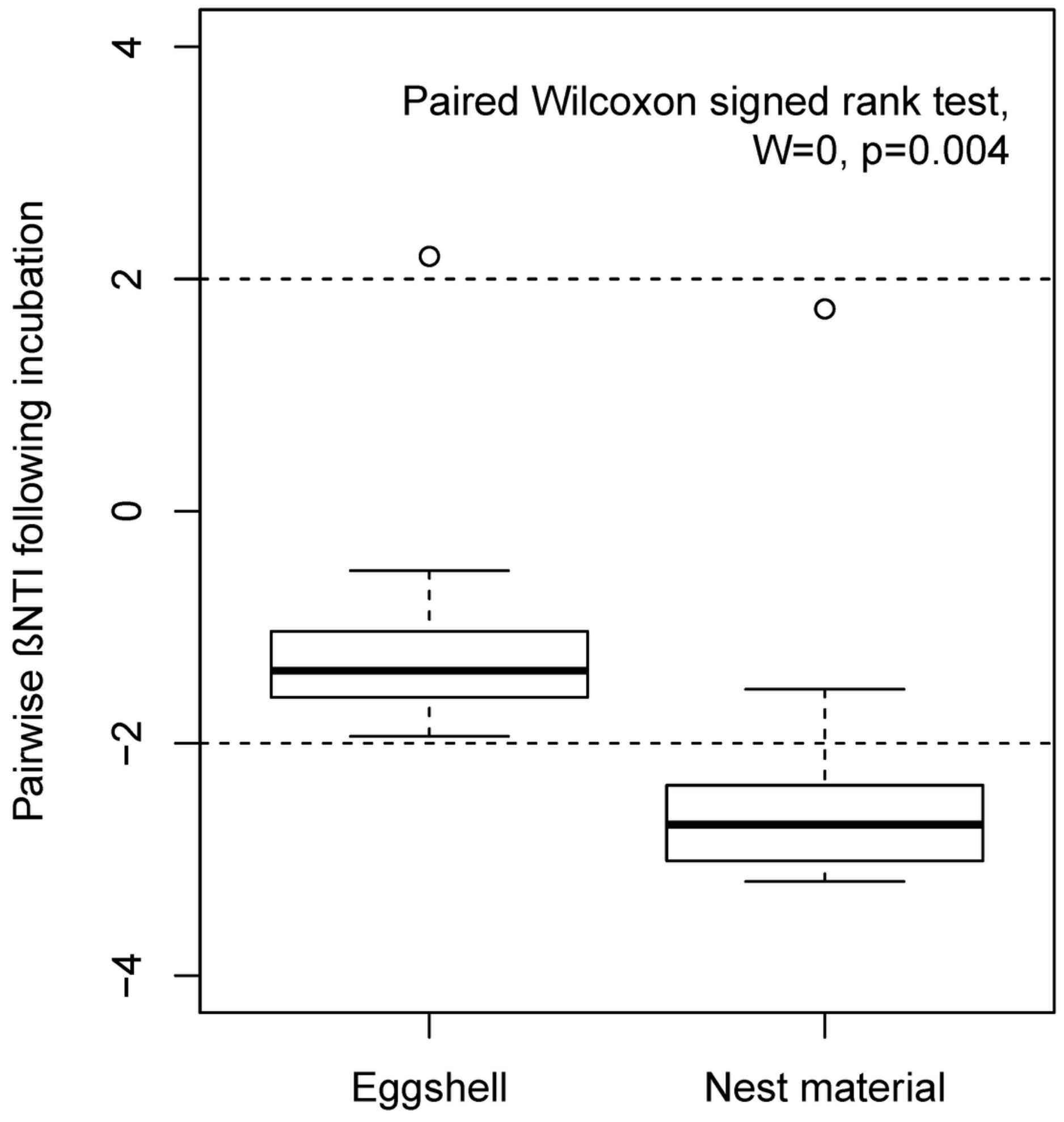

Figure 7

Comparison of $\beta$-NTI ( $\beta$-nearest taxon index) between eggshell and nest material microbiomes in Oriental tit (Parus minor) nests $(n=9$; Table 1$)$.

\section{Supplementary Files}


This is a list of supplementary files associated with this preprint. Click to download.

- Table1.xlsx

- SupplementaryTableS6.xlsx

- Supplementaryinformation.pdf 